\title{
Leishmania infantum induces high phagocytic capacity and intracellular nitric oxide production by human proinflammatory monocyte
}

\author{
Christiana Vargas Ribeiro', Bruna Fonte Boa Rocha', Edward Oliveira1, Andrea Teixeira-Carvalho², \\ Olindo Assis Martins-Filho ${ }^{2}$, Silvane Maria Fonseca Murta ${ }^{1 /+}$, Vanessa Peruhype-Magalhães ${ }^{2}$ \\ ${ }^{1}$ Fundação Oswaldo Cruz-Fiocruz, Instituto René Rachou, Grupo de Genômica Funcional e Proteômica de Leishmania spp. \\ e Trypanosoma cruzi, Belo Horizonte, MG, Brasil \\ ${ }^{2}$ Fundação Oswaldo Cruz-Fiocruz, Instituto René Rachou, Grupo Integrado de Pesquisas em Biomarcadores, Belo Horizonte, MG, Brasil
}

BACKGROUND The mechanism of resistance to $\mathrm{Sb}^{\text {III }}$ in Leishmania is complex, multifactorial and involves not only biochemical mechanisms, but also other elements, such as the immune system of the host.

OBJECTIVES In this study, putative changes in the immunological profile of human monocytes infected with wild-type (WT) and antimony ( $\mathrm{Sb}^{\mathrm{III}}$ )-resistant Leishmania (Viannia) braziliensis and Leishmania (Leishmania) infantum lines were evaluated.

METHODS Susceptibility assays WT and SbIII-resistant L. braziliensis and L. infantum were performed using lines THP-1 human monocytic lineage. Phagocytic capacity, cytokine profile, intracellular nitric oxide (NO) production and surface carbohydrate residues profile were performed in peripheral blood monocytes by flow cytometry.

FINDINGS The phagocytic capacity and intracellular NO production by classical $\left(\mathrm{CD} 14^{++} \mathrm{CD} 16^{-}\right)$and proinflammatory $\left(\mathrm{CD}_{1} 4^{++} \mathrm{CD} 16^{+}\right)$monocytes were higher in the presence of $L$. infantum lines compared to L. braziliensis lines. The results also highlight proinflammatory monocytes as the cellular subpopulation of major relevance in a phagocytosis event and NO expression. It is important to note that $L$. infantum induced a proinflammatory cytokine profile characterised by higher levels of TNF- $\alpha$ in culture supernatant than L. braziliensis. Conversely, both Leishmania lines induce high levels of IL-6 in culture supernatant. Analysis of the expression profile of surface carbohydrates showed that L. braziliensis presents 4.3 -fold higher expression of galactose $(\beta 1,4) N$-acetylglucosamine than $L$. infantum line. Interestingly, the expression level of $\alpha$ - $N$-acetylgalactosamine residues was 2 -fold lower in the $\mathrm{Sb}^{\mathrm{III}}$-resistant $L$. braziliensis line than its counterpart WT line, indicating differences in surface glycoconjugates between these lines.

MAIN CONCLUSIONS Our results showed that L. braziliensis and L. infantum induce different innate immune responses and a highly inflammatory profile, which is characteristic of infection by L. infantum, the species associated with visceral disease.

Key words: L. braziliensis - L. infantum - monocyte subsets - lectins - cytokines - nitric oxide - surface glycoconjugates

Leishmaniasis is caused by over 21 different species of unicellular protozoan parasites belonging to the genus Leishmania. It is classified as a neglected tropical disease, according to the World Health Organization (WHO), and is a public health issue in many developing countries. ${ }^{(1)}$ Leishmaniasis is characterised by a spectrum of clinical manifestations ranging from selfhealing cutaneous (CL) and mucocutaneous (MCL) skin lesions to a visceral (VL) form, which is lethal if untreated. In the New World, L. (L.) infantum (syn. L. (L.) chagasi) is the causative agent of VL, whereas $L$. (V.) braziliensis causes CL and MCL. ${ }^{(2)}$

Many studies have been carried out to identify genes that may be involved in the N-methylglucamine antimoniate (Glucantime ${ }^{\circledR}$ )-resistance phenotype, first-choice

doi: 10.1590/0074-02760190408

Financial support: CNPq, FAPEMIG (CBB-PPM00610/15), CAPES.

EO, ATC, SMFM and BFBR are research fellows supported by CNPq; VPMP by FAPEMIG; CVR by CAPES.

+ Corresponding author: silvane@cpqrr.fiocruz.br

(D) http://orcid.org/0000-0002-8523-2155

Received 31 October 2019

Accepted 14 February 2020 drug for the treatment of human leishmaniasis in many countries. ${ }^{(3,4)}$ Among the mechanisms of drug resistance in Leishmania spp., the main one is the decrease in drug concentration in parasite. ${ }^{(5)}$ In addition, changes in the surface glycoconjugates expression profile of parasites have also been related to resistance and for this evaluation, lectins, which are glycoproteins of non-immunological origin, that interact in a reversible and specific way to carbohydrates or glycoconjugates are used. Studies involving an analysis of the surface glycoconjugates expression profile using specific lectins revealed that antimony-resistant $L$. donovani clinical isolates from patients in Bihar overexpress terminal glycoconjugates of $N$-acetyl-D-galactosamine residues. ${ }^{(6,7)}$

The mechanism of resistance to $\mathrm{Sb}^{\mathrm{III}}$ in Leishmania is complex, multifactorial and involves not only biochemical mechanisms, but also other elements, such as the immune system of the host. A study using an isolate of L. infantum, with in vitro induced resistance to $\mathrm{Sb}^{\mathrm{III}}$, reported resistance to NO and the cytotoxic effects of activated macrophages. ${ }^{(8)}$ Another study demonstrated that L. braziliensis promastigotes isolated from refractory patients are generally less susceptible to nitric oxide (NO). ${ }^{(9)}$ Moura et al. ${ }^{(10)}$ observed that $\mathrm{Sb}^{\mathrm{III}}$-resistant isolates of $L$. infantum showed a higher rate of infectivity in macrophages and higher resistance to $\mathrm{Sb}^{\mathrm{III}}$ and $\mathrm{NO}$ in vitro compared 
to wild-type (WT) samples. However, the involvement of the host immune system in the mechanisms of antimonyresistance in Leishmania requests to be better elucidated.

Clinical aspects and cure of leishmaniasis are related to the development of an effective and balanced immune response in the host. The intracellular parasite must be contained, whereas the immune response must be strictly controlled to avoid further tissue damage..$^{(11,12)}$

The characteristics of the immunological responses developed during Leishmania sp. infections are quite variable among the affected individuals, resulting in several clinical aspects. These natural differences arise from factors such as the host's immune status, the Leishmania species, parasite exposure, co-infections, among others. ${ }^{(11,12)}$

Immunity against Leishmania is mediated through a complex immunological parameters network, including the innate and adaptive immune response. The immune response plays an important role in the clinical cure of the disease or its progression. ${ }^{(11)}$ Thus, the presence of effector cells such as monocytes/macrophages, is critical for the control or development of leishmaniasis. . $^{(12)}$

Monocytes/macrophages are antigen presenting cells that represent one of the first steps in the innate immune response aimed at killing the Leishmania parasite. These cells use important mechanisms that modulate inflammatory response activation and kill the parasite by producing cytokines as TNF- $\alpha$, and NO, reactive oxygen intermediates (ROI). ${ }^{(13,14)}$

Interestingly, Leishmania spp. modulates monocytes/ macrophage functions by preventing oxidative burst and the effector functions that lead to its elimination and by improvement a bursting microenvironment of modulatory IL-4, IL-10, and TGF- $\beta$ cytokines. ${ }^{(14,15,16,17,18)}$

A better understanding of the immune response against Leishmania spp. is very important to establishing a rational approach to chemotherapy and to comprehend mechanism of drug resistance. In this context, the objective of this study was to analyse changes in the immunological profile of monocytes after WT and $\mathrm{Sb}^{\mathrm{III}}$ resistant $L$. braziliensis and $L$. infantum lines infection and evaluate the agglutination pattern these different parasite species by applying tests that assess the lectins' binding specificity.

\section{SUBJECTS AND METHODS}

Human subjects - The study population consisted of 10 non-infected volunteers. The individuals were five men and five women, ranging from 20 to 31 years of age. They did not report previous infection by Leishmania spp. All individuals live in Belo Horizonte, Minas Gerais, Brazil, and voluntarily agreed to donate blood samples for the research.

Leishmania samples and culture conditions - In this study, we used promastigote forms of Leishmania (Viannia) braziliensis (MHOM/BR/1975/2904) and Leishmania (Leishmania) infantum (MHOM/BR/1974/PP75) lines WT and resistant to potassium antimony tartrate $\left(\mathrm{Sb}^{\mathrm{III}}\right)$. The $\mathrm{Sb}^{\mathrm{III}}$-resistant lines were previously selected in vitro by step-wise increase of $\mathrm{Sb}^{\mathrm{III}}$ drug pressure. ${ }^{(19)}$
The parasites were grown at $26^{\circ} \mathrm{C}$ in M199 medium supplemented with $2 \mathrm{mM}$ L-glutamine, $5 \mu \mathrm{g} / \mathrm{mL}$ hemin, 50 $\mu \mathrm{g} / \mathrm{mL}$ streptomycin, $2 \mu \mathrm{g} / \mathrm{mL}$ biopterin, $1 \mu \mathrm{g} / \mathrm{mL}$ biotin, $40 \mathrm{mM}$ HEPES $\mathrm{pH}$ 7.4, $50 \mathrm{U} / \mathrm{mL}$ penicillin, and $10 \%$ $\mathrm{v} / \mathrm{v}$ heat-inactivated fetal calf serum. All experiments were carried out with parasites in the stationary phase.

Genotyping of Leishmania species using Hsp70 and ITS1 markers - The WT and Sb ${ }^{\mathrm{III}}$-resistant $L$. braziliensis and L. infantum lines were submitted to polymerase chain reaction-restriction fragment length polymorphism (PCR-RFLP) for genotyping analysis using specific primers for HSP70 and ITS1 genes, as previously described. ${ }^{(20)}$ Briefly, genomic DNA of these lines was obtained using the QIAquick DNA Extraction Kit (Qiagen), according to the manufacturer's recommendations. After amplification, the PCR products were digested with the restriction endonuclease enzyme HaeIII to analyse restriction fragments length polymorphisms (RFLP). Restriction profiles were analysed on $4 \%$ agarose GelRed (Biotium) stained and compared to the standard reference Leishmania species.

Susceptibility assays WT and Sb ${ }^{I I I}$-resistant L. braziliensis and L. infantum lines - THP-1 human monocytic lineage (ATCC TIB 202) were cultured in a complete RPMI-1640 medium [supplemented 10\% foetal bovine serum (FBS), $2 \mathrm{mM}$ glutamine, $100 \mathrm{U} / \mathrm{mL}$ penicillin, and $100 \mu \mathrm{g} / \mathrm{mL}$ streptomycin] and differentiated into macrophages by adding $20 \mathrm{ng} / \mathrm{mL}$ phorbol myristate acetate (PMA) in culture. Macrophages $\left(4 \times 10^{5}\right.$ cells/well) were seeded in 24 well cell culture plates containing $13 \mathrm{~mm}$ round coverslips and incubated for $72 \mathrm{~h}$ at $37^{\circ} \mathrm{C}$ in a humid atmosphere containing $5 \% \mathrm{CO}_{2}$. Then, the adhered macrophages were exposed to promastigote forms of WT and $\mathrm{Sb}^{\mathrm{III}}$-resistant $L$. braziliensis and L. infantum lines at the stationary phase $\left(4 \times 10^{6}\right.$ parasites/well $)(10: 1$ parasites/ macrophage). After $5 \mathrm{~h}$ of infection, the free parasites were removed and a RPMI-1640 medium was added in the absence or presence of $\mathrm{Sb}^{\mathrm{III}}$ at a concentration ranging from 12.5 to $200 \mu \mathrm{M}$. After $72 \mathrm{~h}$ of incubation, the adhered macrophages were stained by panoptic staining. The infected cells and the number of intracellular amastigotes were determined using ImageJ software ( $1.50 \mathrm{i}$ version, Wayne Rasband National Institute of Health). $\mathrm{EC}_{50}$ values were obtained from three independent measurements in triplicate, using the linear interpolation method.

L. braziliensis and L. infantum Alexa Fluor 647-labelling procedures - Promastigote forms of WT and

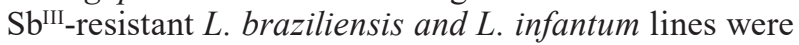
obtained from cultures in a M199 medium supplemented with $10 \%$ FBS, maintained at $26^{\circ} \mathrm{C}$ in BOD incubator for five and seven days, respectively. The parasites were stained with Alexa Fluor 647 at a final concentration of $3.2 \mu \mathrm{g} / \mathrm{mL}$ at $37^{\circ} \mathrm{C}$ for $45 \mathrm{~min}$ in a $5 \% \mathrm{CO}_{2}$ incubator. After the Alexa Fluor 647-labelling procedure, parasites were washed once with phosphate buffered saline (PBS) pH 7.4 and resuspended in PBS supplemented with $10 \%$ FBS. The Alexa Fluor 647-labelled parasite suspension was adjusted to $1 \times 10^{8} / \mathrm{mL}$ and maintained at $26^{\circ} \mathrm{C}$ in a BOD incubator until use. Aliquots of Alexa Fluor 
647-labelled parasites were run in LSR Fortessa (BD) to evaluate the efficiency of the Alexa Fluor 647-labelling procedure. The ideal Alexa Fluor 647-labelled parasite staining would lead to a single peak around $10^{3}$ and $10^{4}$ log intervals in histogram plots.

In vitro L. braziliensis and L. infantum promastigote peripheral blood monocyte infection - Human heparinised whole blood was centrifuged at $800 \mathrm{xg}$ for $10 \mathrm{~min}$ at $18^{\circ} \mathrm{C}$. The plasma was removed, the whole blood cells (erythrocytes and leukocytes) washed two times with RPMI 1640 medium, and the final cell suspension adjusted to $1 \times 10^{7}$ cells $/ \mathrm{mL}$. For the Alexa Fluor 647-labelled promastigotes platform, $1 \times 10^{7}$ leukocytes were incubated with $1 \mathrm{~mL}$ of RPMI 1640 medium and $50 \mu \mathrm{L}$ of Alexa Fluor 647-labelled WT or $\mathrm{Sb}^{\mathrm{III}}$-resistant L. infantum or L. braziliensis promastigotes at $5 \times 10^{6}$ parasites $/ \mathrm{mL}$. Short-term in vitro cultures were performed in five distinct platforms for promastigote forms referred to as "uninfected culture", "infected WT promastigote of $L$. infantum culture", "infected $\mathrm{Sb}^{\mathrm{III}}$-resistant promastigote of $L$. infantum culture", "infected WT promastigote of L. braziliensis culture", and "infected $\mathrm{Sb}^{\mathrm{III}}$-resistant promastigote of L. braziliensis culture" by $5 \mathrm{~h}$ of incubation under gentle shaking in an orbital shaker, at $37^{\circ} \mathrm{C}$ in a $5 \% \mathrm{CO}_{2}$ incubator. After incubation, each tube was centrifuged at $600 \mathrm{xg}$ for $7 \mathrm{~min}$ at $18^{\circ} \mathrm{C}$. The supernatants were maintained for 1 month at $-70^{\circ} \mathrm{C}$ for cytokine analysis.

Intracellular NO expression in peripheral blood monocytes after WT and Sb ${ }^{I I I}$-resistant L. braziliensis and L. infantum in vitro infection - The NO expression was performed using peripheral blood monocytes as previously reported. ${ }^{(14,21)}$ Membrane-permeable fluorescent NO indicator, DAF-2DA is deacetylated by intracellular esterases to DAF-2 that reacts with NO to yield the highly fluorescent, a membrane-impermeable compost, the triazolofluorescein (DAF-2T), allow to distinguish between NO generated inside the monocytes and NO-derived from an exogenous source. The culture was performed using $1 \times 10^{7}$ leukocytes incubated with $5 \mu \mathrm{L}$ of WT or $\mathrm{Sb}^{\mathrm{III}}$-resistant $L$. braziliensis or $L$. infantum Alexa Fluor 647-labelled promastigotes for $1 \mathrm{~h}$ at $37^{\circ} \mathrm{C}$ in a $5 \% \mathrm{CO}_{2}$ incubator. A control culture ("uninfected culture") was used to determine the basal levels of intracellular NO. The samples were then incubated in the presence of 4,5-diaminofluorescein diacetate (DAF-2DA) $(10 \mu \mathrm{M}$, Sigma, MO, USA) for $3 \mathrm{~h}$ at $37^{\circ} \mathrm{C}$ in a $5 \% \mathrm{CO}_{2}$ incubator. The samples were labelled for $20 \mathrm{~min}$ at room temperature in the presence of PerCP-labelled anti-CD16 mAb, V450-labelled anti-CD14 $\mathrm{mAb}$ and PE-labelled anti-HLA-DR mAb. Following incubation, the red blood cells were lysed with FACS lysing solution (Becton Dickinson, CA, USA) and the cell pellet was washed and centrifuged at $600 \mathrm{x} g$ for 7 min at room temperature and resuspended with PBS solution for immediate acquisition in the LSR Fortessa cytometer. A total of 3,000 events/tube on $\mathrm{CD} 14^{+}$monocyte gate was acquired. In order, to evaluate the enzymatic origin of the monocytes NO detected, the selective iNOS inducer [lipopolysaccharide (LPS) $10 \mu \mathrm{g} / \mathrm{mL}$, Sigma, USA] and inhibitor (Aminoguanidine-AG 10mM, Sigma, MO, USA) were used. The LPS and AG cultures were used as functional specificity controls associated with monocytes NO production by iNOS pathway in all experiments. The data were analysed using Flow Jo 10.1 software.

Detection of supernatant cytokine levels by cytometric bead array - The cytometric bead array (CBA) is a method in which the simultaneous measurement of multiple cytokines is performed in a single sample. In this study, culture supernatant aliquots were used to quantify the secreted cytokine levels using CBA Human Inflammatory kit (Becton Dickinson, San Diego, CA, USA) for evaluation of IL-8, IL-1b, IL-6, IL-10, TNF-a, IL-12 p70 (limit of detection: $3.6 \mathrm{pg} / \mathrm{mL}, 7.2 \mathrm{pg} / \mathrm{mL}, 2.5 \mathrm{pg} / \mathrm{mL}, 3.3$ $\mathrm{pg} / \mathrm{mL}, 3.7 \mathrm{pg} / \mathrm{mL}$ and $1.9 \mathrm{pg} / \mathrm{mL}$, respectively). Briefly, $25 \mu \mathrm{L}$ of supernatants or standards were added to $17 \mu \mathrm{L}$ of mixture beads and incubated for $3 \mathrm{~h}$ at room temperature in the dark. After incubation, the samples and standards were washed with $500 \mu \mathrm{L}$ of wash buffer and centrifuged at $600 \mathrm{x} \mathrm{g}$ for $7 \mathrm{~min}$ at room temperature. Subsequently, $17 \mu \mathrm{L}$ of detection cocktail consisting of mix PE-conjugated $\mathrm{mAbs}$ were added to each tube and the mixture reincubated for $180 \mathrm{~min}$ at room temperature in the dark. Following incubation, the samples and standards were washed again with $500 \mu \mathrm{L}$ of wash buffer and centrifuged at $600 \mathrm{x} \mathrm{g}$ for $7 \mathrm{~min}$ at room temperature. After washing, $250 \mu \mathrm{L}$ of wash buffer were added to each tube prior to data acquisition (300 events/tube) using FACSVerse flow cytometer (Becton Dickinson, San Diego, CA, USA). Data analysis was performed using FCAP Array 3.1 software (Becton Dickinson, San Diego, CA, USA). Secreted cytokines concentrations were expressed as $\mathrm{pg} / \mathrm{mL}$ and the results plotted in bar graphs presenting mean + standard error of the mean (SEM) in log scale.

Expression profile of surface carbohydrates using Erythrina cristagalli, Dolichos biflorus, and Concanavalin A lectins in WT and Sb ${ }^{I I I}$-resistant L. braziliensis and L. infantum lines - The Erythrina cristagalli (ECA), Dolichos biflorus (DBA), and Concanavalin-A (Con-A) lectins were used to compare the expression profile of the surface carbohydrates in WT and antimony-resistant $L$. braziliensis and $L$. infantum lines, as they have different carbohydrate binding specificities. ECA binds to residues of galactose $(\beta 1,4) N$-acetylglucosamine, DBA recognises $\alpha-N$-acetylgalactosamine residues, and Con-A binds to terminal portions of $\alpha$-D-mannose and $\alpha$-D-glucose residues. Promastigote forms were incubated separately with each of these lectins conjugated to fluorescein isothiocyanate (FITC). Briefly, promastigote forms of the WT and $\mathrm{Sb}^{\text {III-}}$-resistant $L$. braziliensis and $L$. infantum lines in the stationary growth phase $(2 \mathrm{x}$ $10^{6}$ parasites $/ \mathrm{mL}$ ) were washed with PBS and incubated with each lectin conjugated to FITC: ECA and DBA (EY Laboratories, INC, San Mateo, CA, USA) and Con A (Vector Laboratories, Burlingame, CA, USA) at a final concentration of $10 \mathrm{mg} / \mathrm{mL}$ for $30 \mathrm{~min}$ at $37^{\circ} \mathrm{C}$ in a $5 \%$ $\mathrm{CO}_{2}$ incubator. The total of 30.000 parasites/tube were then acquired to flow cytometer (LSR Fortessa) and the data were analysed using Flow Jo v.10 software. The geometric mean fluorescence intensity (gMFI) and the Con-A labelled-Leishmania percentage (\%) for each $L$. braziliensis and L. infantum sample was determined. 
A

Leishmania spp. infection profile in THP-1 macrophages after Sb"t treatment
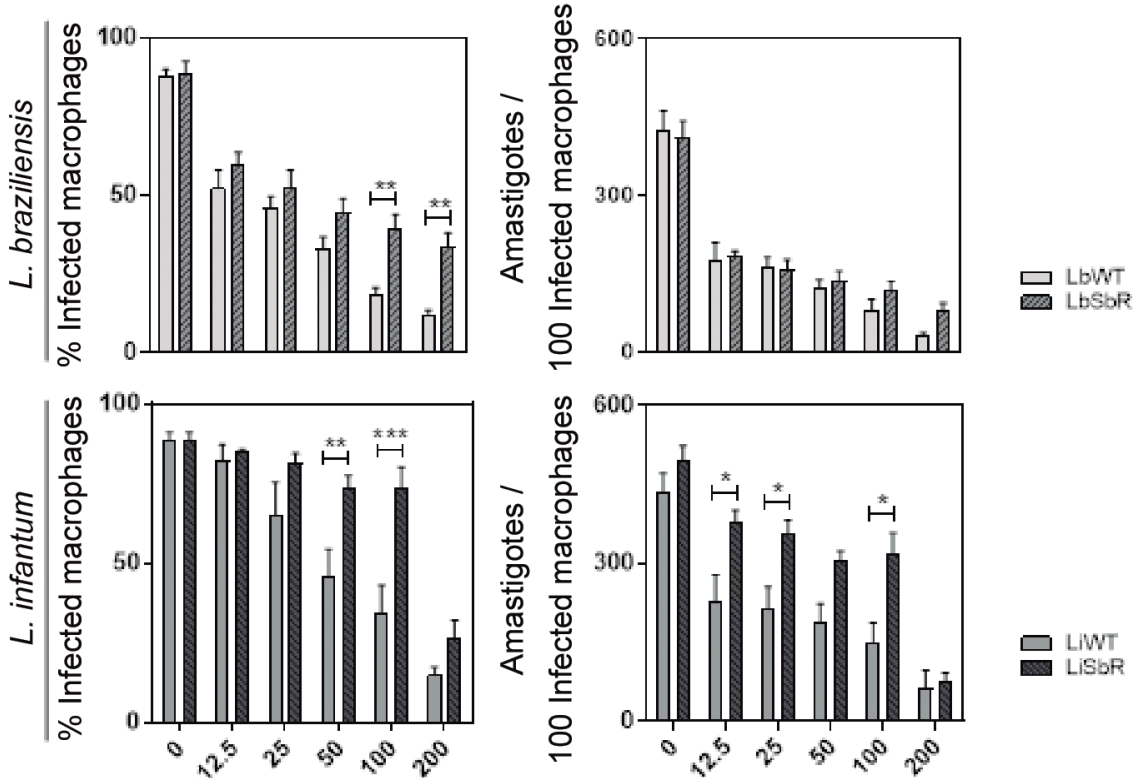

$\left[\mathrm{Sb}^{\mathrm{II}}\right] \mu \mathrm{M}$

C

$\mathrm{Sb}^{\|}$EC50 of wild-type and resistant $L$. braziliensis and $L$. infantum lines

\begin{tabular}{ccc}
\hline & Sb $^{\text {III }} \boldsymbol{\mu M}$ & Fold \\
\hline L. braziliensis WT & 17.0 & \\
L. braziliensis SbR & 34.8 & 2.05 \\
L. infantum WT & 50.2 & \\
L. infantum SbR & 133.3 & 2.65 \\
\hline
\end{tabular}

Fig. 1: $\mathrm{Sb}^{\text {III }}$ susceptibility was evaluated on amastigote forms using THP-1-derived-human macrophages. (A) THP-1 macrophages infected with wild-type (WT) (LbWT) and Sb ${ }^{\text {III }}$-resistant (LbSbR) Leishmania braziliensis lines (top graph) or WT (LiWT) and Sb ${ }^{\text {III }}$-resistant (LiSbR) L. infantum lines (bottom graph) were cultured in the absence or presence of increasing Sb ${ }^{\text {III }}$ concentrations $(12.5$ to $200 \mu \mathrm{M})$ for $72 \mathrm{~h}$ and the

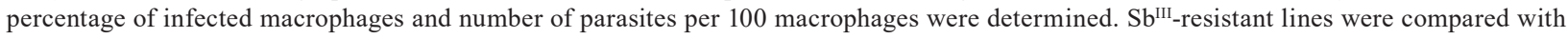
their respective WT counterparts and the statistically significant differences $(* \mathrm{p}<0.05, * * \mathrm{p}<0.01, * * * \mathrm{p}<0.001)$ were represented by connector lines. (B) Resistance index of the $\mathrm{EC}_{50}$ value for LbSbR and LiSbR compared to the $\mathrm{EC}_{50}$ value for LbWT and LiWT.

Statistical analysis - Statistical analyses were performed using GraphPad Prism software (San Diego, USA, version 5.00). First, the Kolmogorov-Smirnov test was performed as normality test to evaluate the Gaussian distribution of data. The unpaired $t$ test was used for comparative analyses between two groups, whereas, the one-way analysis of variance followed by Tukey's posttest were used for comparative analyses among three or more groups. For susceptibility assays, bidirectional analysis of variance (ANOVA two-way) followed by the Bonferroni post-test was used. In all cases, the differences were considered statistically significant when the $p$ value was less than 0.05 .

Ethics - This work complied with resolution number 466/2012 from the National Health Council for research involving human subjects and was approved by the Ethical Committee at Instituto René Rachou (CEPSH/ IRR/FIOCRUZ protocol:1.368.058), Belo Horizonte, Minas Gerais, Brazil.

\section{RESULTS}

Genotyping of Leishmania lines using $H s p 70$ and ITS1 markers - The ITS1 and Hsp70 molecular markers for genotyping were used to confirm the Leishmania species used in this study, of WT and $\mathrm{Sb}^{\mathrm{III}}$-resistant $L$. braziliensis and L. infantum lines. Both pairs of Hsp70 and ITS1 primers amplified fragments of 1300 and 300350 bp, respectively, in all Leishmania species analysed. The restriction profiles of the PCR products were compared to the standard reference Leishmania species: 
A

Analysis strategies of phagocytic capacity in monocytes subpopulation
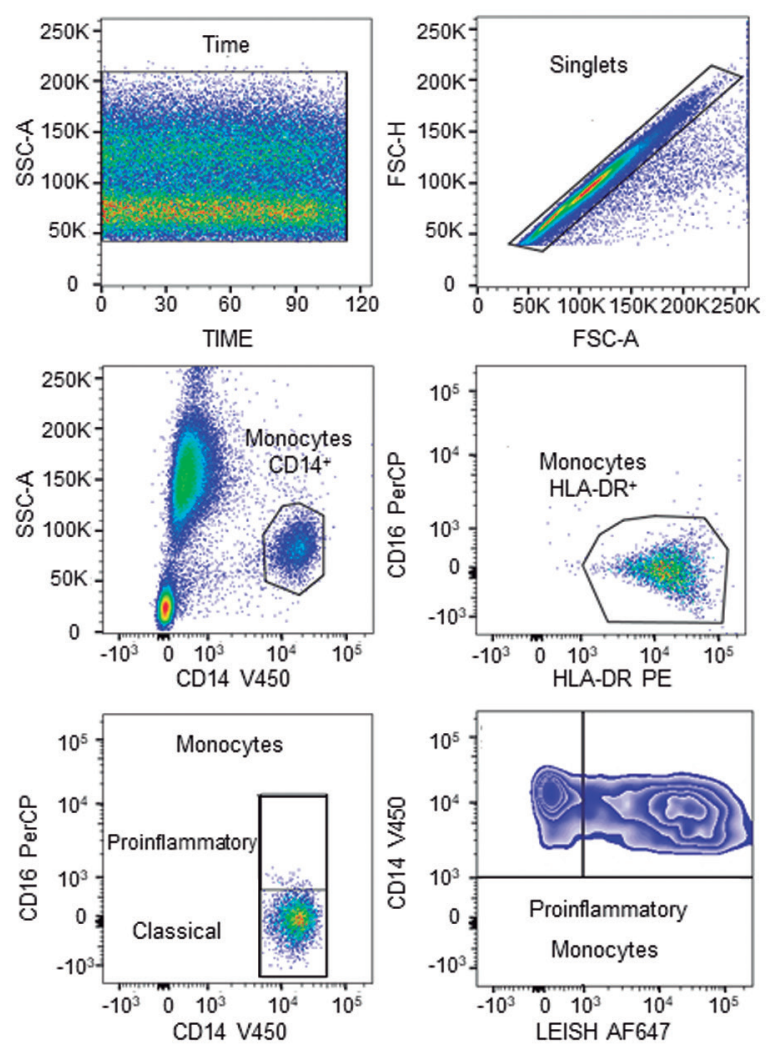

B

Phagocytic capacity in different monocytes subpopulation

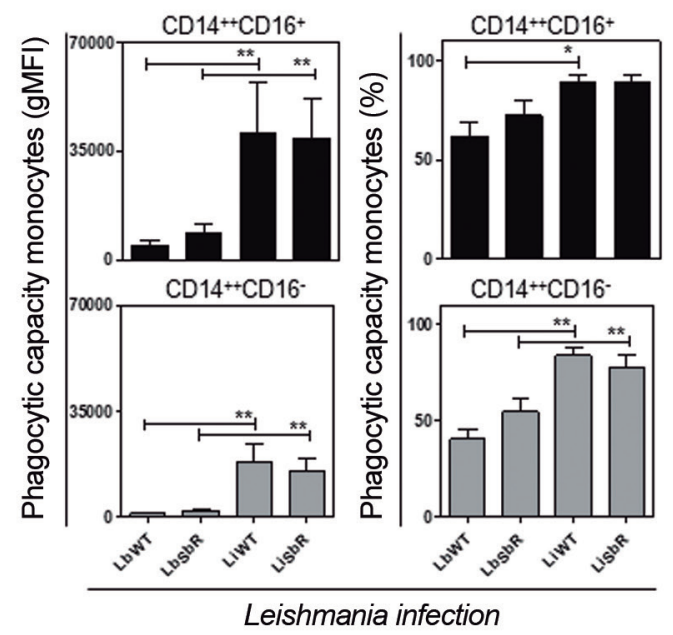

C

Phagocytic capacity in proinflammatory versus classical monocytes

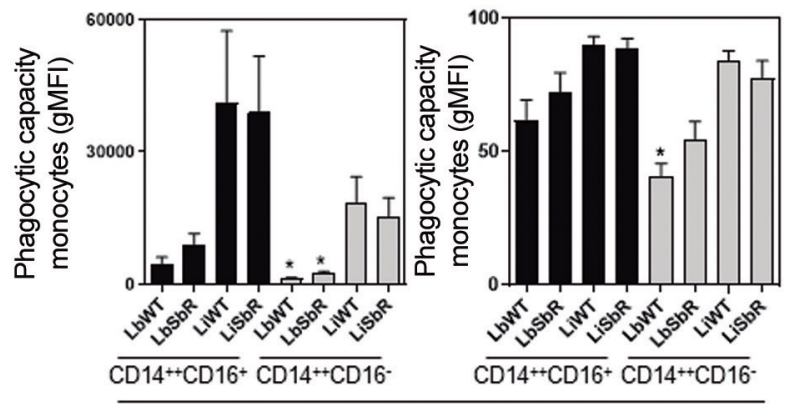

Leishmania infection

Fig. 2: (A) flow cytometric analysis of monocyte subpopulations. First, the quality of the acquisition was estimated in Time versus Side ScatterA graphs (SSC-A, granularity). Then, the selection of singlets was performed in Forward Scatter (FSC-A, size) versus FSC-H. Discrimination of the monocyte population was possible in anti-CD14-V450 versus SSC-A plot. Classical $\left(\mathrm{CD} 14^{++} \mathrm{CD} 16\right)$ and proinflammatory $\left(\mathrm{CD} 14^{++} \mathrm{CD} 16^{+}\right)$ monocyte subpopulations were plotted in anti-CD14-V450 versus anti-CD16-PerCP graphs, and the evaluation of the phagocytic capacity was obtained in anti-Leishmania AF647 ${ }^{+}$versus anti-CD14-V450 graphs to classical $\left(\mathrm{CD} 14^{++} \mathrm{CD} 16\right)$ and proinflammatory $\left(\mathrm{CD} 14^{++} \mathrm{CD} 16^{+}\right)$monocyte subpopulations. An illustrative graph of phagocytic capacity of proinflammatory $\left(\mathrm{CD} 14^{++} \mathrm{CD} 16^{+}\right)$monocyte was presented in the last image. (B) Analysis of the phagocytic capacity of $\mathrm{CD} 14^{++} \mathrm{CD} 16^{-}$and $\mathrm{CD} 14^{++} \mathrm{CD} 16^{+}$monocytes of healthy volunteers were shown in bar graphs presenting mean + SEM, after incubation with wild-type (WT) and $\mathrm{Sb}^{\mathrm{III}}$-resistant L. braziliensis and L. infantum lines. The results were presented by percentage (\%) and geometric mean fluorescence intensity (gMFI); statistically significant differences $(* P<0.05$, $* * P<0.01)$ between WT

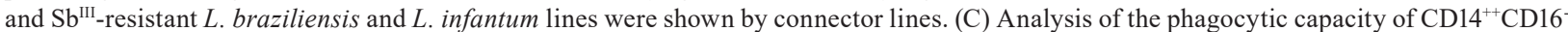
versus $\mathrm{CD}_{1}{ }^{++} \mathrm{CD} 6^{+}$monocytes of healthy volunteers were shown in bar graphs presenting mean $+\mathrm{SEM}$, after incubation with $\mathrm{WT}_{\text {and }} \mathrm{Sb}^{\mathrm{II}}$ resistant $L$. braziliensis and $L$. infantum lines. Statistically significant differences $(P<0.05)$ between the promastigote of $\mathrm{WT}$ and $\mathrm{Sb}^{\mathrm{III}}$-resistant Leishmania lines in different monocyte subpopulations were shown by asterisks $\left(^{*}\right)$.

L. amazonensis (IFLA/BR/67/PH8), L. braziliensis (MHOM/BR/75/M2903), L. infantum (MHOM/BR/74/ PP75), and L. guyanensis (MHOM/BR/75/M4147). The results confirmed that the Leishmania species used in this work correspond to L. braziliensis and L. infantum [Supplementary data (Figure)].

Susceptibility assays of WT and Sb ${ }^{I I I}$-resistant L. braziliensis and L. infantum lines using THP-1 macrophages - To analyse whether the $\mathrm{Sb}^{\mathrm{III}}$-resistant phenotype persists in parasite intracellular forms, amastigote forms of WT and $\mathrm{Sb}^{\mathrm{III}}$-resistant L. braziliensis and L. infantum lines were submitted to susceptibility assays with $\mathrm{Sb}^{\mathrm{III}}$. The data showed that the numbers of infected macrophages were higher in the

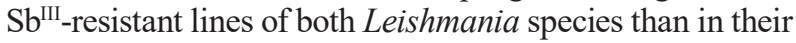
WT counterparts (Fig. 1A). The number of amastigotes/100 infected macrophages was also higher in the $\mathrm{Sb}^{\text {III-resistant }}$ than in the WT L. infantum line. The amastigote forms from both $\mathrm{Sb}^{\mathrm{III}}$-resistant $L$. braziliensis and $L$. infantum lines were about 2.05 and 2.65-fold more resistant to $\mathrm{Sb}^{\mathrm{III}}\left(\mathrm{EC}_{50}\right.$ 34.8 and $133.3 \mu \mathrm{M}$ for LbSbR and LiSbR, respectively) than to their respective counterpart WT lines $\left(\mathrm{EC}_{50} 17\right.$ and 50.2 $\mu \mathrm{M}$ for LbWT and LiWT) (Fig. 1B).

Phagocytic capacity of proinflammatory and classical monocytes - In order to evaluate the phagocytic ca- 
A
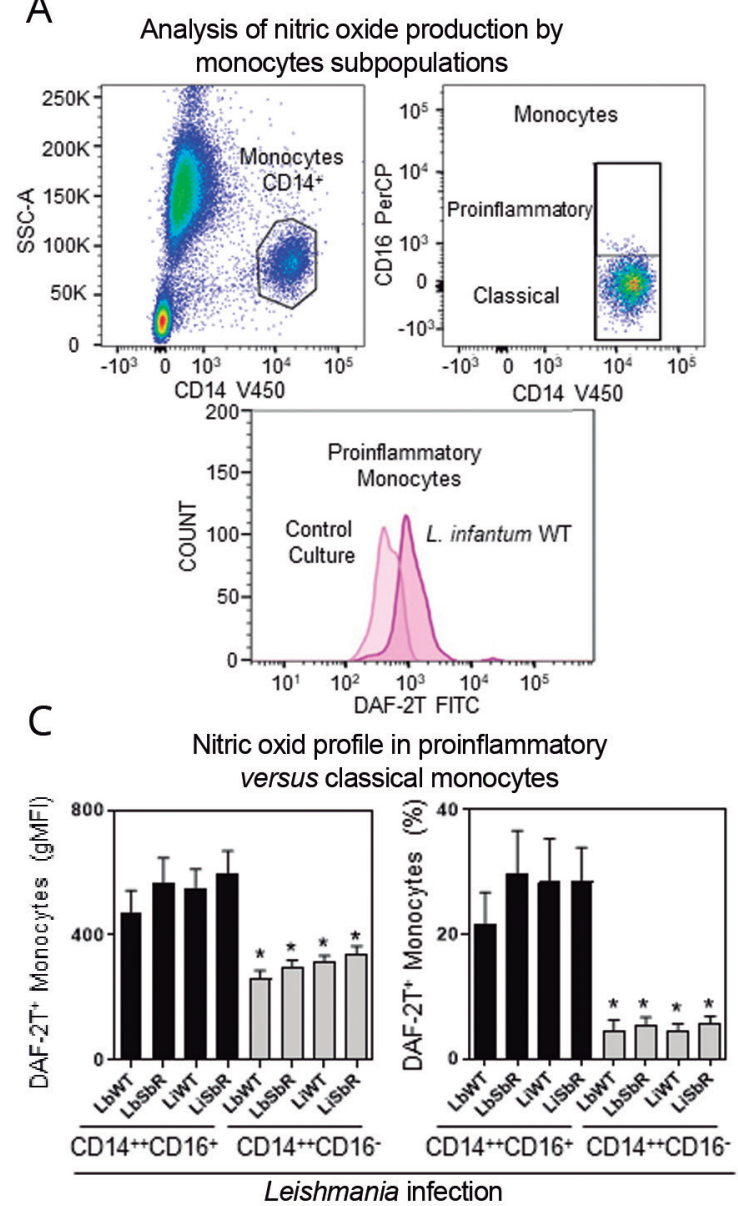

B

Nitric oxid profile after Leishmania spp. infection
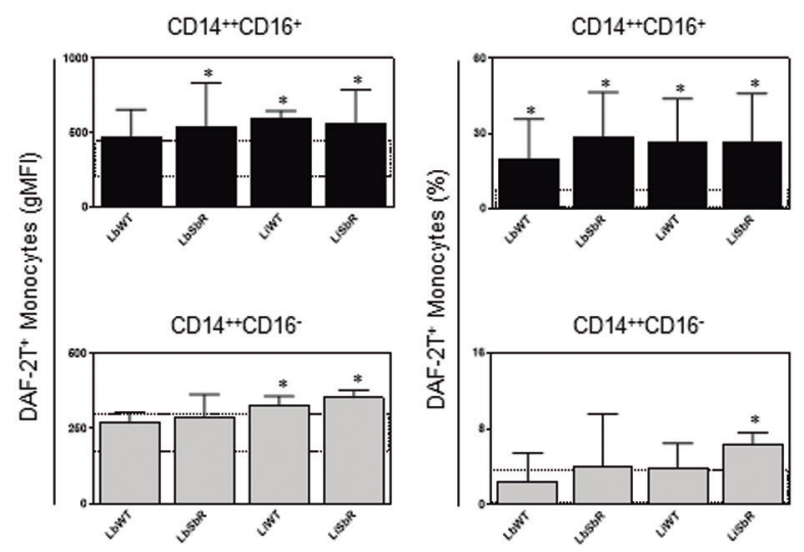

D

Proinflammatory and modulatory cytokines profile

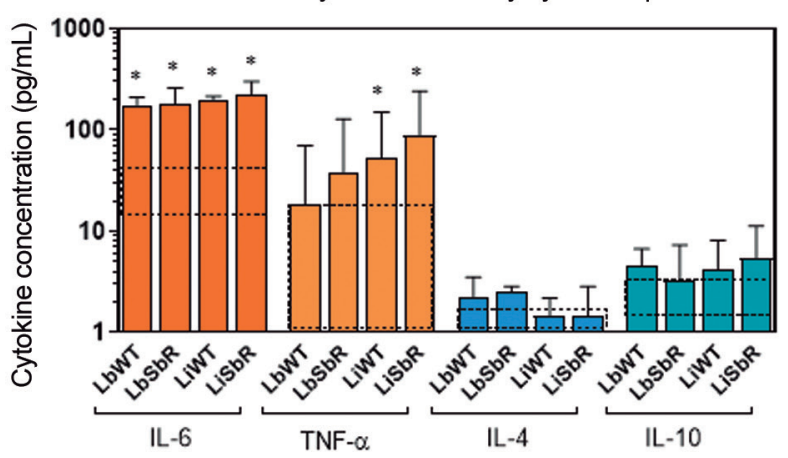

Fig. 3: (A) nitric oxide (NO) strategy analysis in monocytes. Discrimination of the monocyte population was possible in anti-CD14-V450 versus SSC-A plot. Classical $\left(\mathrm{CD} 14^{++} \mathrm{CD} 16\right)$ and proinflammatory $\left(\mathrm{CD} 14^{++} \mathrm{CD} 16^{+}\right)$monocyte subpopulations were plotted in anti-CD14-V450 versus anti-CD16PerCP graphs, and the intracellular NO expression was obtained in a DAF-2T FITC versus count histogram. (B) Analysis of the intracellular NO expression by $\mathrm{CD} 14^{++} \mathrm{CD} 16^{-}$and $\mathrm{CD} 14^{++} \mathrm{CD} 16^{+}$monocytes of healthy volunteers were shown in bar graphs presenting mean $+\mathrm{SEM}$, after incubation with promastigotes of wild-type (WT) and $\mathrm{Sb}^{\mathrm{II}}$-resistant Leishmania braziliensis and L. infantum lines. The control culture (basal NO expression) was shown by dashed rectangle (----) presenting the $95 \%$ confidence interval $(95 \% \mathrm{CI})$. Statistically significant differences $(\mathrm{p}<0.05)$ between promastigote of WT and $\mathrm{Sb}^{\mathrm{III}}$-resistant Leishmania lines as compared to control culture were shown by asterisks $(*)$. (C) Analysis of the intracellular NO expression by $\mathrm{CD} 14^{++} \mathrm{CD} 16-$ versus $\mathrm{CD} 14^{++} \mathrm{CD} 16^{+}$monocytes of healthy volunteers were shown in bar graphs presenting mean + SEM, after incubation with promastigotes of WT and SbIII-resistant $L$. braziliensis and $L$. infantum lines. The results were presented by percentage (\%) and geometric mean fluorescence intensity $(\mathrm{gMFI})$. Statistically significant differences $(\mathrm{p}<0.05)$ between promastigotes of WT and Sb ${ }^{\text {III }}$-resistant Leishmania lines in different monocyte subpopulations were identified by asterisks $\left(^{*}\right)$. (D) Secreted cytokine profile was performed in culture supernatant of whole blood after promastigotes of WT and $\mathrm{Sb}^{\mathrm{III}}$-resistant $L$. braziliensis and $L$. infantum lines infection using flow cytometric CBA immunoassay. Secreted IL-6, TNF-a, IL-4, and IL-10 concentrations were expressed as $\mathrm{pg} / \mathrm{mL}$ and the results plotted in bar graphs presenting mean + SEM in log scale. The control culture (basal secreted cytokines) was shown by dashed rectangle (----) presenting the $95 \% \mathrm{CI}$. Statistically significant differences $(\mathrm{p}<0.05)$ between promastigotes of WT and $\mathrm{Sb}^{\mathrm{III}}$-resistant Leishmania lines cultures and control culture were shown by asterisks $\left(^{*}\right)$.

pacity (percentage of monocytes Leish ${ }^{+}$) of peripheral blood proinflammatory/ $\left(\mathrm{CD} 14^{++} \mathrm{CD} 16^{+}\right)$and classical $\left(\mathrm{CD} 14^{++} \mathrm{CD} 16^{-}\right)$monocytes, they were infected with WT and $\mathrm{Sb}^{\text {III }}$-resistant $L$. braziliensis and L. infantum lines and evaluated by flow cytometry using phenotypicspecific selection strategy (anti-HLA-DR PE, anti-CD14 V450, and anti-CD16 PerCP antibodies) (Fig. 2A). The results showed that the phagocytic capacity of proinflammatory and classical monocytes was higher for WT and $\mathrm{Sb}^{\mathrm{III}}$-resistant $L$. infantum lines as compared to $L$. braziliensis lines (Fig. 2B). Moreover, our data high- light proinflammatory monocytes as the cellular subpopulation of major relevance in a phagocytosis event (Fig. 2C). A second approach was used as complementary analysis with the purpose to evaluate the infection density of the monocytes (gMFI of infected cells). Our data demonstrated that despite the high percentage of $L$. braziliensis $^{+}$proinflammatory monocytes (above 50\% of infected cells), the number of parasites internalised or present in the cell membrane (interaction) is small as compared to L. infantum challenge (Fig. 2B - top graphs). On the other hand, no differences were found 
A

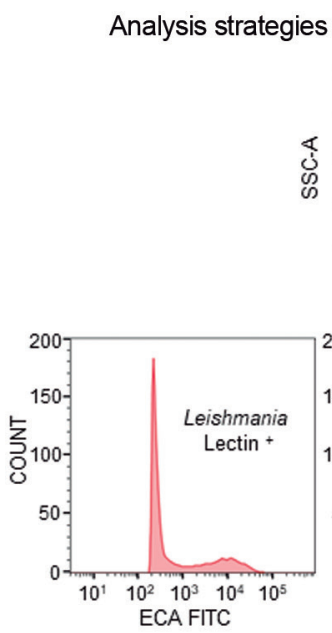

C

Expression pattern of ECA, DBA and CON-A by wild-type and $\mathrm{Sb}^{\text {"II }}$ resistant Leishmania lines

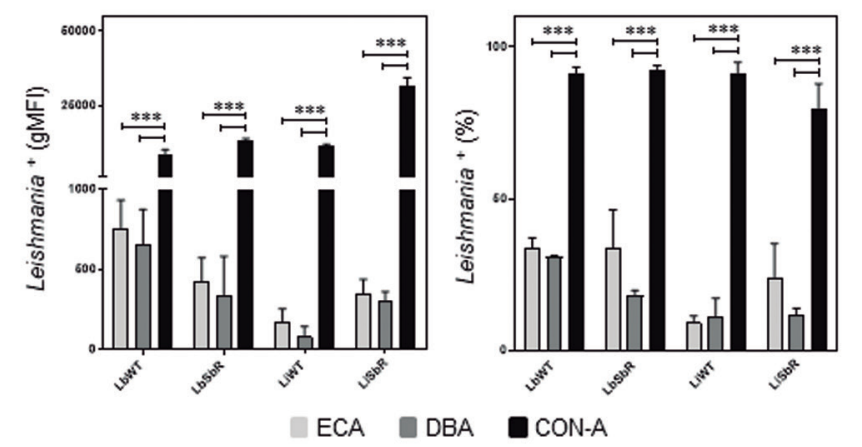

B

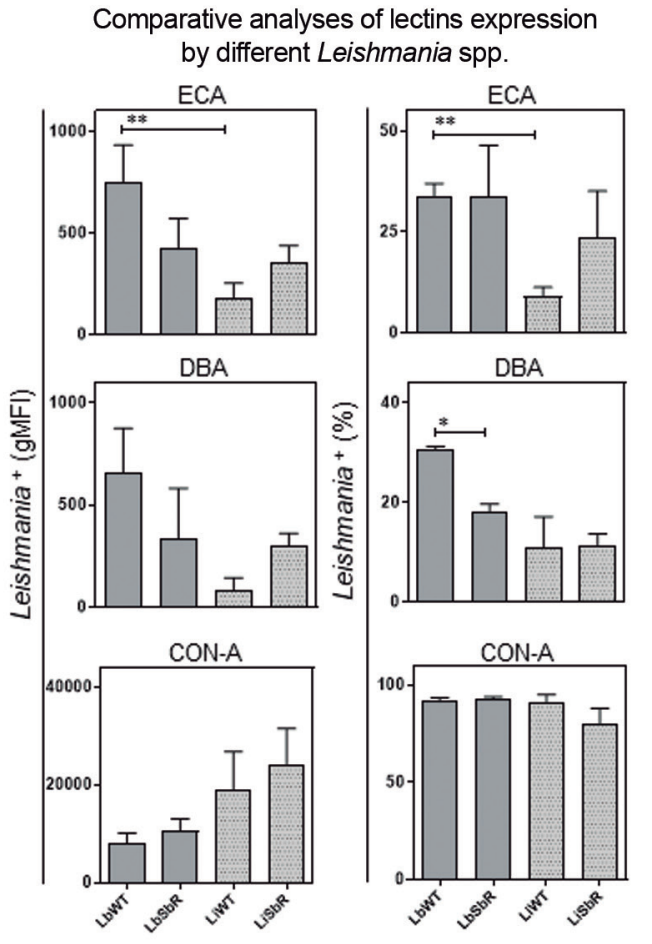

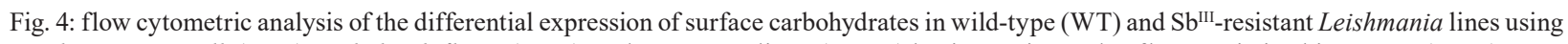
Erythrina cristagalli (ECA), Dolichos biflorus (DBA), and Concanavalin-A (Con-A) lectins conjugated to fluorescein isothiocyanate (FITC). ECA binds to galactose $(\beta 1,4) N$-acetylglucosamine residues, DCA recognises $\alpha$ - $N$-acetylgalactosamine residues, and Con-A binds to terminal portions of $\alpha$-D-mannose and $\alpha$-D-glucose residues. (A) The Leishmania spp. population was selected in SSC-A versus FSC-A graph, and ECA, DBA and Con-A lectin profiles were performed in lectin-FITC versus count histograms. (B) Comparison of the ECA, DBA, and Con-A lectins binding profiles were presented in bar graphs showing the mean $+\mathrm{SEM}$ for WT and Sb ${ }^{\mathrm{III}}$-resistant L. braziliensis and L. infantum lines. The results were presented by percentage (\%) and geometric mean fluorescence intensity (gMFI), and statistically significant differences $(* \mathrm{p}<0.05 ; * * \mathrm{p}<0.01)$ between $\mathrm{Sb}^{\mathrm{III}}$-resistant $L$. braziliensis and L. infantum lines were shown by connector lines. (C) Evaluation of the ECA, DBA, and Con-A lectins binding profiles for each Leishmania spp. line was presented in bar graphs showing the mean + SEM. Statistically significant differences $(* * * p<$ 0.001) between Con-A expression and the other lectins in WT and Sb ${ }^{\mathrm{III}}$-resistant $L$. braziliensis and L. infantum lines were shown by connector lines.

between $\mathrm{Sb}^{\mathrm{III}}$-resistant parasites as compared to WT of the same Leishmania spp.

\section{Functional biomarkers evaluation after WT and $\mathrm{Sb}^{\mathrm{II}}$-resistant promastigote $L$. braziliensis and $L$. infantum infection}

Intracellular $N O$ expression by proinflammatory and classical monocytes - The reagent 4,5-diaminofluorescein diacetate (DAF-2DA) was used to evaluate intracellular NO expression. The monocytes were evaluated by flow cytometry in anti-CD14 V450 versus anti-CD16 PerCP plots, and NO expression was evaluated in histogram graphs highlighting the displacement of DAF-2T FITC in X axis (Fig. 3A). NO inducer (LPS) and NO inhibitor (AG) stimuli were used as functional and specificity controls, respectively. The control culture and WT and $\mathrm{Sb}^{\mathrm{III}}$-resistant L. braziliensis and L. infantum promastigote cultures were used to evaluate the basal and Leishmania-specific NO production by proinflammatory and classical monocytes. The LPS data presented mean + SEM equal to $62.29 \pm 8.45(\%$ - proinflammatory monocytes) and $24.23 \pm 8.21$ ( $\%$ - classical monocytes). Already AG analyses showed mean + SEM equal to 6.69 \pm 3.37 (\% -proinflammatory monocytes) and $3.22 \pm 0.35$ (\% - classical monocytes), indicative of perfect protocol controls. Data analyses were performed in comparison with control culture (basal cytokine profile - dashed rectangle presenting the $95 \% \mathrm{CI}$; Fig. 3B). The results 
showed that $\mathrm{Sb}^{\mathrm{III}}$-resistant $L$. braziliensis and both WT and $\mathrm{Sb}^{\mathrm{III}}$-resistant $L$. infantum lines induced a statistically significant increase in fluorescence intensity and percentage of DAF- $2 \mathrm{~T}^{+}$proinflammatory monocytes ( $\mathrm{p}$ $<0.05$; Fig. 3B - Top Graphs). Interestingly, our data demonstrated a significant increase in fluorescence intensity and percentage of classical DAF- $2 \mathrm{~T}^{+}$monocytes subpopulation after L. infantum stimulation (Fig. 3B Bottom Graphs). It also highlighted proinflammatory monocytes as the principal source of NO as compared to classical monocytes (Fig. 3C). No differences were found between $\mathrm{Sb}^{\mathrm{III}}$-resistant parasites as compared to WT of the same Leishmania spp.

Proinflammatory and modulatory cytokine profile in culture supernatant - The Cytometric Bead Array (CBA) system was used to quantify cytokine supernatant levels. Promastigote forms of all Leishmania lines analysed induced high levels of proinflammatory IL-6 cytokine as observed in the supernatants of the different culture conditions. Data analyses were performed in comparison with control culture [basal cytokine profile - dashed rectangle presenting the $95 \%$ confidence interval (95\%CI); Fig.3D]. Interestingly, both WT and $\mathrm{Sb}^{\text {III }}$-resistant $L$. infantum lines also induced higher levels of TNF- $\alpha(p<0.05$; Fig. 3D). The data did not show differentiated expression of modulatory cytokines, IL-4 and IL-10, in the culture conditions (Fig. 3D).

Evaluation of surface carbohydrate residues in $L$. braziliensis and L. infantum lines - We also investigated the expression profile of the surface carbohydrates galactose $(\beta 1,4) N$-acetylglucosamine (ECA), $N$-acetylgalactosamine (DBA), and Concanavalin-A (Con-A) in the WT and $\mathrm{Sb}^{\mathrm{III}}$-resistant lines of each species. The Leishmania parasites were selected in SSC-A versus FSC-A graphs and the surface carbohydrates profile was performed by lectin-FITC expression (Fig. 4A). The results showed that the WT L. braziliensis line (LbWT) expresses 4.3 -fold more galactose $(\beta 1,4) \mathrm{N}$-acetylglucosamine residue compared to the WT $L$. infantum line (LiWT) (ECA-Lectin ${ }^{+}$). In addition, a 2-fold lower expression of $N$-acetylgalactosamine residue (DBA-Lectin $^{+}$) was detected in the $\mathrm{Sb}^{\mathrm{III}}$-resistant $L$. braziliensis as compared to its WT counterpart (Fig. 4B). A similar parasite percentage Con-A-labelled for each Leishmania sample was observed, indicating the homogeneity of the parasites regarding the expression of molecules containing mannose or glucose residues (Fig. 4C). It is important to emphasize that regardless of antimony-resistance phenotype, $\alpha$-D-mannose and $\alpha$-D-glucose are the most prevalent carbohydrates in the membrane of parasites compared to other evaluated.

\section{DISCUSSION}

Parasites of the genus Leishmania appear to be related to escape mechanisms that repress the normal functions of the macrophage, an immune system effector cell. ${ }^{(11,12)}$ Monocytes/macrophages are antigen-presenting cells that act as parasite hosts and are responsible for eliminating them. Phagocytosed parasites are an important mechanism modulating the activation of the inflammatory response and controlling their growth. (11,12) According to the phenotypic-functional properties, the monocytes population is segregated into classical $\left(\mathrm{CD} 14^{++} \mathrm{CD} 16-\right)$, proinflammatory $\left(\mathrm{CD} 14^{++} \mathrm{CD} 16^{+}\right)$and patrolling $\left(\mathrm{CD} 14^{\mathrm{dim}} \mathrm{CD} 16^{+}\right)$monocytes. ${ }^{(22)}$ The classical monocyte subset shows a high expression of genes associated with tissue repair, anti-apoptotic properties, and acts as the first line of defense of the innate immune response against intracellular pathogens due to its phagocytic capacity. Proinflammatory monocytes express cytoskeletal rearrangement genes at high levels, presenting high phagocytosis capacity, anti-proliferative and pro-apoptotic properties, and produce higher levels of proinflammatory cytokines. ${ }^{(23)}$

Previous studies have shown that a good Type $1 \mathrm{im}-$ mune response (proinflammatory profile) is required, aiding in parasite protection. If the response is Type 1, cytokines such as IL-2, IFN-g, TNF-a, and IL-12 will be produced, promoting the activation of macrophages, NO synthesis, and parasite destruction. Conversely, if the response is Type 2, IL-4 and IL-10 will be produced, which cause a low macrophage activation and, consequently, clinical forms will appear. ${ }^{(11,12,24)}$ Several Leishmania species have the ability to suppress the response to infection through inhibition of NO synthesis and proinflammatory cytokine production. ${ }^{(11,12,24)}$ However, recent literature has showed that L. braziliensis-induced infection has been characterised by an inflammatory response. Cytokines such as IL-6, IL-1 $\beta$, IL-17 and TNF-a have been associated with exacerbation of the infection, with TNF-a, an important NO inducer, being particularly significant in mucosal leishmaniasis evolution. ${ }^{(24)}$ In VL, the increased production of inflammatory cytokines without parallel infection control has also contributed to pathogenesis. Several human VL studies have demonstrated elevated serum levels of IL-4, IL-6, IL-10, IL-12, IL-13, IFN-g, and TNF-a in active disease compared to asymptomatic infection..$^{(15,16,25)}$ In this sense, the progression/worsening of the clinical status would be promoting a lack of control of the inflammatory response and this "cytokine storm" without modulation favors the systemic inflammatory syndrome in VL patients and disfiguring mucosal lesions. ${ }^{(25,26)}$ On the other hand, as in other infectious and parasitic diseases, individuals who development efficient immunological response to the infection and do not presented the apparent disease allows to understand the immunological mechanisms of protection against infection. These individuals present satisfactory balance between modulatory and inflammatory biomarkers. In Tegumentary Leishmaniasis the subclinical infection is characterised by a lower IFN-g and TNF-a production, suggesting that this modulated immune response has the ability to control infection without causing pathology. ${ }^{(27)}$ Similarly, asymptomatic VL individuals presented basal profile cytokine. ${ }^{(15,16,25)}$

In this study, the main objective was to analyse changes in the immunological profile of human peripheral blood monocytes after infection with WT and $\mathrm{Sb}^{\mathrm{III}}$ resistant $L$. braziliensis and $L$. infantum lines. Our data demonstrated that $L$. infantum induced higher levels of TNF-a compared to L. braziliensis. However, both Leish- 
mania species induce high levels of IL-6 in monocyte culture supernatants. Our results showed that $L$. infantum induced a higher phagocytic capacity, associated with NO and TNF-a production by human monocytes as compared to L. braziliensis. However, our group, in cutaneous leishmaniasis, showed protective immune response associate with CD23-IgE-mediated NO release, iNOS specific and increase of intracellular NO after treatment. ${ }^{(14,18)}$ Thus, our data are in concordance with literature studies.

Regarding cytokine profile, although we not determine the source, we believed that the monocytes were the main cell population associated with this cytokine profile because they are physiologically the peripheral blood cells that most contribute with TNF-a and IL-6 production. Our data of proinflammatory monocytes reinforce this hypothesis since proinflammatory monocytes are highlighted as the cellular subpopulation of greater relevance in TNF-a and IL-6 production. In our study, we observed increase of Leishmania phagocytosis, specially L. infantum and NO expression and suggest that the monocytes are the main responsible by the TNF-a and IL-6 production.

This interspecific difference showing that $L$. infantum induced greater phagocytic capacity and production of NO and TNF-a by human monocytes compared to L. braziliensis. In the literature, studies point to the existence of polymorphisms in the structure of LPG intra and interspecies of Leishmania. ${ }^{(28,29)}$ Polymorphisms of the glycoconjugates (LPG, GIPLs and gp63), are important in the differential regulation of the initial events of the immune response, as well as in the establishment of the infection. The variation in LPG structure results in a differential activation of macrophages, resulting in distinct production of cytokines and/or NO levels. ${ }^{(29)}$ The presence of genetic diversity among lines allows a distinct ability to infect macrophages and thus the parasite surface molecules can modulate the interaction between Leishmania and host cells, influencing the clinical course of the disease $\mathrm{e}^{(30)}$ and on the appearance of different immunopathology in leishmaniasis. ${ }^{(28)}$ As mentioned, discrepancies in the structure and composition of these molecules can influence the innate immune response and the differential establishment of the infection, generating a variety of clinical manifestations, such as LV and LT.(28)

Interesting, no difference was observed in the phagocytic capacity and $\mathrm{NO}$ /cytokine production by human monocytes between WT and $\mathrm{Sb}^{\mathrm{III}}$-resistant L. braziliensis and L. infantum lines. The resistant lines studied here have a low antimony-resistance index (2.0 and 2.65-fold) in THP-1 macrophages. We believe that if this resistance index had been higher, it would possibly alter the phagocytic capacity and other complementary parameters evaluated in the study. The $\mathrm{Sb}^{\mathrm{III}}$-resistance mechanism in Leishmania is complex, multifactorial, and involves not only biochemical mechanisms but also other elements, such as the host immune system. Literature data using other antimony-resistant models have revealed some differences in Leishmania lines. Holzmuller et al. ${ }^{(8)}$ reported that an isolate of $L$. infantum with resistance induced in vitro to $\mathrm{Sb}^{\mathrm{III}}$ was resistant to $\mathrm{NO}$ donors and the cytotoxic effects of activated macrophages. It has also been shown that $L$. braziliensis promastigotes isolated from refractory patients are generally less susceptible to NO ${ }^{(9)}$ Moura et al. ${ }^{(10)}$ observed that antimony-resistant $L$. infantum isolates had a higher rate of infectivity in macrophages and a greater resistance to $\mathrm{Sb}^{\mathrm{III}}$ and $\mathrm{NO}$ in vitro as compared to WT samples. The authors further suggested that clinical isolates of $L$. infantum resistant to $\mathrm{Sb}^{\text {III }}$ may induce the production of inflammatory cytokines and inhibit the mechanisms associated with macrophage death.

We also investigated the expression profile of the surface carbohydrates galactose $(\beta 1,4) N$-acetylglucosamine, using Erythrina cristagalli lectin (ECA), and $N$ acetylgalactosamine, using Dolichos biflorus (DBA) in the WT and $\mathrm{Sb}^{\mathrm{III}}$-resistant L. braziliensis and L. infantum lines. The data showed a higher expression of galactose $(\beta 1,4) \mathrm{N}$-acetylglucosamine in WT L. braziliensis than in $L$. infantum line, indicating interspecific differences of the surface carbohydrates. The DBA expression profile showed that the $\mathrm{Sb}^{\text {III }}$-resistant $L$. braziliensis line expresses lower $\alpha$ - $N$-acetylgalactosamine residues than its counterpart WT line. In contrast, Mukhopadhyay et al., ${ }^{(7)}$ using the same ECA and DBA lectins, showed that antimony-resistant $L$. donovani isolates overexpress surface glycoconjugates containing $N$-acetylgalactosaminyl residues. This difference may be due to different Leishmania species analysed. Drug resistance mechanism is complex, multifactorial and varies among the different Leishmania species analysed. The role of these surface glycoconjugates in the antimony-resistance phenotype should be further investigated. Concanavalin-A was used as a control and our data also showed the homogeneity of parasite populations in both species regarding the expression of molecules containing mannose and glucose residues, possibly LPG, since approximately $90 \%$ of the parasites were labelled with Con-A.

In this study, we used promastigote forms of only one strain of each Leishmania species: L. braziliensis (2904) and L. infantum (PP75), then further studies analysing a greater number of strains are need to support better the results.

In conclusion, our results showed that the phagocytic capacity and intracellular NO and TNF-a cytokine production by human monocytes were higher in the presence of $L$. infantum lines as compared to L. braziliensis lines. In addition, the data also highlighted proinflammatory monocytes as the cellular subpopulation of major relevance in phagocytosis events and $\mathrm{NO}$ expressions.

\section{ACKNOWLEDGEMENTS}

To the Program for Technological Development in Tools for Health-PDTIS-FIOCRUZ for use of its facilities.

\section{AUTHORS' CONTRIBUTION}

CVR, BFBR, SMFM and VPM designed, performed the experiments and analysed the data; CVR, BFBR, EO, ATC, OAMF, SMFM and VPM wrote and reviewed the full manuscript. All authors read and approved the final manuscript. The authors declare that there is no conflict of interests.

\section{REFERENCES}

1. WHO - World Health Organization: Leishmaniasis. Available from: lhttp://www.who.int/en/news-room/fact-sheets/detail/leishmaniasis. Accessed Sep 102018. 
2. Pearson RD, Sousa AQ. Clinical spectrum of leishmaniasis. Clin Infect Dis. 1996; 22(1): 01-13.

3. Croft SL, Sundar S, Fairlamb AH. Drug resistance in leishmaniasis. Clin Microbiol Rev. 2006; 19(1): 111-26.

4. Mukherjee A, Boisvert S, Monte-Neto RL, Coelho AC, Raymond F, Mukhopadhyay R, et al. Telomeric gene deletion and intrachromosomal amplification in antimony-resistant Leishmania. Mol Microbiol. 2013; 88(1): 189-202.

5. Jeddi F, Piarroux R, Mary C. Antimony resistance in Leishmania, focusing on experimental research. J Trop Med. 2011; 2011(43): 1-15.

6. Mukhopadhyay R, Mukherjee S, Mukherjee B, Naskar K, Mondal $\mathrm{D}$, Decuypere S, et al. Characterization of antimony-resistant Leishmania donovani isolates: biochemical and biophysical studies and interaction with host cells. Int J Parasitol. 2011; 41(13-14): 1311-21.

7. da Silva LCN, Correia MTS. Plant lectins and Toll-like receptors: implications for therapy of microbial infections. Front Microbiol. 2014; 5(1): 20-3.

8. Holzmuller P, Sereno D, Lemesre JL. Lower nitric oxide susceptibility of trivalent antimony-resistant amastigotes of Leishmania infantum. Antimicrob Agents Chemother. 2005; 49(10): 4406-9.

9. Souza AS, Giudice A, Pereira JMb, Guimaraes LH, de Jesus AR, Moura TR, et al. Resistance of Leishmania (Viannia) braziliensis to nitric oxide: correlation with antimony therapy and TNF-alpha production. BMC Infect Dis. 2010; 15(10): 209-19.

10. Moura TR, Santos MLB, Braz JM, Santos LFVC, Aragão MT, de Oliveira FA, et al. Cross-resistance of Leishmania infantum isolates to nitric oxide from patients refractory to antimony treatment, and greater tolerance to antileishmanial responses by macrophages. Parasitol Res. 2016; 115(2): 713-21.

11. Liese J, Schleider U, Bogdan C. The innate immune response against Leishmania parasites. Immunobiology. 2008; 213(3-4): 377-87.

12. Stafford JL, Neumann NF, Belosevic M. Macrophage-mediated innate host defense against protozoan parasites. Crit Rev Microbiol. 2002; 28(3): 187-248.

13. de Assis Souza M, de Castro MCAB, de Oliveira AP, de Almeida AF, de Almeida TM, Reis LC, et al. Cytokines and NO in American tegumentary leishmaniasis patients: profiles in active disease, after therapy and in self-healed individuals. Microb Pathog. 2013; 57(4): 27-32.

14. Carvalho-Gontijo R, Peruhype-Magalhães V, Costa-Silva MF, Martins-Filho OA, Quaresma PF, Freire JM, et al. Protective profile involving CD23/IgE-mediated NO release is a hallmark of cutaneous leishmaniasis patients from the Xakriabá indigenous community in Minas Gerais, Brazil. Scand J Immunol. 2015; 81(6): 515-24.

15. Peruhype-Magalhães V, Martins-Filho OA, Prata A, Silva LA, Rabello A, Teixeira-Carvalho A, et al. Immune response in human visceral leishmaniasis: analysis of the correlation between innate immunity cytokine profile and disease outcome. Scand J Immunol. 2005; 62(5): 487-95.

16. Peruhype-Magalhães V, Martins-Filho OA, Prata A, Silva LA, Rabello A, Teixeira-Carvalho A, et al. Mixed inflammatory/ regulatory cytokine profile marked by simultaneous raise of interferon-gamma and interleukin-10 and low frequency of tumour necrosis factor-alpha $(+)$ monocytes are hallmarks of active human visceral Leishmaniasis due to Leishmania chagasi infection. Clin Exp Immunol. 2006; 146(1): 124-32.
17. Nylén S, Gautam S. Immunological perspectives of leishmaniasis. J Glob Infect Dis. 2010; 2(2): 135-46.

18. Rodrigues-Alves ML, Melo-Júnior OAO, Coelho-Dos-Reis JG, Pascoal-Xavier MA, Alves-Costa H, Reis CA, et al. Fc $\gamma$-RI, Fc $\gamma$ RII and IL-10 as predictive biomarkers for post-therapeutic cicatrization time in monocytes from cutaneous leishmaniasis patients. Parasite Immunol. 2018; 40(8): 01-12.

19. Liarte DB, Murta SMF. Selection and phenotype characterization of potassium antimony tartrate-resistant populations of four New World Leishmania species. Parasitol Res. 2010; 107(1): 205-12.

20. Schönian G, Nasereddin A, Dinse N, Schweynoch C, Schallig $\mathrm{HDFH}$, Presber W, et al. PCR diagnosis and characterization of Leishmania in local and imported clinical samples. Diagn Microbiol Infect Dis. 2003; 47(1): 349-58.

21. Gomes JAS, Campi-Azevedo AC, Teixeira-Carvalho A, SilveiraLemos D, Vitelli-Avelar D, Sathler-Avelar R, et al. Impaired phagocytic capacity driven by downregulation of major phagocytosis-related cell surface molecules elicits an overall modulatory cytokine profile in neutrophils and monocytes from the indeterminate clinical form of Chagas disease. Immunobiology. 2012; 217(10): 1005-16.

22. Antonelli LRV, Leoratti FMS, Costa PAC, Rocha BC, Diniz SQ, Tada MS, et al. The CD14+CD16+ inflammatory monocyte subset displays increased mitochondrial activity and effector function during acute Plasmodium vivax malaria. PLoS Pathog. 2014; 10(9): 01-16.

23. Wong KL, Tai JJ-Y, Wong W-C, Han H, Sem X, Yeap W-H, et al. Gene expression profiling reveals the defining features of the classical, intermediate, and nonclassical human monocyte subsets. Blood. 2011; 118(5): 16-31.

24. Boaventura VS, Santos CS, Cardoso CR, de Andrade J, dos Santos WLC, Clarêncio J, et al. Human mucosal leishmaniasis: neutrophils infiltrate areas of tissue damage that express high levels of Th17-related cytokines. Eur J Immunol. 2010; 40(10): 2830-6.

25. Costa ASA, Costa GC, de Aquino DMC, de Mendonça VRR, Barral A, Barral-Netto M, et al. Cytokines and visceral leishmaniasis: a comparison of plasma cytokine profiles between the clinical forms of visceral leishmaniasis. Mem Inst Oswaldo Cruz. 2012; 107(6): 735-9.

26. Costa DL, Rocha RL, Carvalho RMA, Lima-Neto AS, Harhay $\mathrm{MO}$, Costa CHN, et al. Serum cytokines associated with severity and complications of Kala-Azar. Pathog Glob Health. 2013; 107(2): 78-87.

27. Novoa R, Bacellar O, Nascimento M, Cardoso TM, Ramasawmy $\mathrm{R}$, Oliveira WN, et al. IL-17 and regulatory cytokines (IL-10 and IL-27) in L. braziliensis infection. Parasite Immunol. 2011; 33(2): 132-6.

28. Assis RR, Ibraim IC, Nogueira PM, Soares RP, Turco SJ. Glycoconjugates in New World species of Leishmania: polymorphisms in lipophosphoglycan and glycoinositolphospholipids and interaction with hosts. Biochim Biophys Acta. 2012; 1820(9): 1354-65.

29. Ibraim IC, Assis RR, Pessoa NL, Campos MA, Melo MN, Turco $\mathrm{SJ}$, et al. Two biochemically distinct lipophosphoglycans from Leishmania braziliensis and Leishmania infantum trigger different innate immune responses in murine macrophages. Parasit Vectors. 2013; 6(3): 54-64.

30. Farias LHS, Rodrigues APD, Silveira FT, Seabra SH, DaMatta RA, Saraiva EM, et al. Phosphatidylserine exposure and surface sugars in two Leishmania (viannia) braziliensis strains involved in cutaneous and mucocutaneous leishmaniasis. J Infect Dis. 2013; 207(3): 537-43. 